Asociación de Jóvenes Historiadores y Arqueólogos de Murcia

PANTR REL

REVISTA DE CIENCIA

$$
\begin{array}{r}
Y \\
\text { DIDÁCTICA } \\
\text { DE LA HISTORIA } \\
\text { III - } 2^{2} \text { época }
\end{array}
$$




\section{ASOCIACIÓN DE JÓVENES HISTORIADORES Y ARQUEÓLOGOS DE MURCIA}

\section{PANTA REI. REVISTA DE CIENCIA Y DIDÁCTICA \\ DE LA HISTORIA III. $2^{a}$ época}


CONSEJO EDITORIAL: LAURA ARIAS FERRER

ALEJANDRO EGEA VIVANCOS

ANTONINO GONZÁLEZ BLANCO

RAFAEL GONZÁLEZ FERNÁNDEZ

JOAQUÍN LOMBA MAURANDI

ENRIQUE QUINTANA CIFUENTES

GONZALO MATILLA SÉIQUER

JOSÉ ANTONIO MOLINA GÓMEZ

REDACTOR JEFE:

ÁNGEL LUIS GONZÁLEZ TORRES

Depósito legal: MU-966-1995

I.S.S.N. 1136-2464

Edición de Compobell, S.L. Murcia 


\section{ÍNDICE}

AGRADECIMIENTOS

Presentación

Por Alejandro Egea Vivancos, Laura Arias Ferrer

\section{ARTÍCULOS}

Análisis microscópico de la industria lítica: la traceología

Por Ignacio Martín Lerma

Reflexiones en torno al estudio de la economía en Prehistoria

Por Valentín Martínez García

Aplicaciones SIG en el análisis de las sociedades del pasado. Un caso de estudio: Las primeras comunidades campesinas del Levante Peninsular

Por Gabriel García Atiénzar

La situación actual de los estudios de egiptología en España

Por José Javier Martínez García

La vida cotidiana en el Imperio Asirio. Usos y costumbres de un pueblo que conquistó las Cuatro Regiones del Mundo

Por Ángel Luis González Torres

Las formas de intercambio y las estructuras comerciales orientalizantes en la Vega Baja del Segura: dos variables de estudio arqueológico

Por Sara Pernas García 
Evolución de los patrones de asentamiento en época ibérica. Una propuesta de estudio del mundo ibérico murciano a través del análisis del poblamiento

Hispania Tierra de Roma. Organización y gestión del suelo

Por $M^{a}$ Carmen Santapau Pastor

Hacia una arqueología de la España bizantina. Breves notas a propósito del seminario Work in progress

Por Jaime Vizcaíno Sánchez

Reflexiones acerca de un estudio sobre las estancias auxiliares en la arquitectura barroca catedralicia

Por Francisca del Baño Martínez

La investigación sobre fisiognomía y expresión de las pasiones. Objetivos y metodología

Por María del Mar Albero Muñoz 


\title{
REFLEXIONES EN TORNO AL ESTUDIO DE LA ECONOMÍA EN PREHISTORIA
}

\author{
VALENTÍN Martínez García
}

\section{Resumen}

Es nuestro objetivo, dentro de la investigación histórica, la comprensión de las sociedades, como éstas se organizaban y se administraban a sí mismas. Consideramos, como pretendemos dejar patente en las líneas que componen este artículo, que la economía es la expresión más social de una sociedad, y que por tanto, el análisis de la economía dada a estudio es la vía más apropiada para aproximarnos a su conocimiento.

En este artículo desglosaremos que se ha de entender por economía, dejando atrás viejas concepciones y guiándonos por la ciencia económica más actual, además de esbozar en el mismo algunos de los interrogantes y reflexiones que debemos plantearnos para conocer el sistema económico de las sociedades a estudio.

Palabras clave: Elección, escasez, población, propiedad, trabajo, productividad, enriquecimiento, intercambio, especialización.

\begin{abstract}
It is our goal, in historical research, to understand societies, how those were organized and administered themselves. We believe, as we want to make clear in the lines of this paper, that the economy is the most social expression of a society and, therefore, the analysis of a given economy is the most appropriate way to approach to its knowledge.

In this paper we disentangle what economy is, abandoning old ideas and guiding us by the current economic science; in addition to outline at the same time some of
\end{abstract}


the questions and thoughts that we need to ponder to understand the economic system of the societies being studied.

Key Words: Choice, scarcity, population, property, work, productivity, enrichment, exchange, specialization.

\section{El origen de la palabra economía}

El origen de la palabra Economía viene del griego 'Oikos Nemo', que significa la casa bien administrada. Serían los griegos los primeros en hablar de economía. Mas en concreto, fue Jenofonte (427-355 a.c.) el primer autor que hablaría de economía en su obra el «económico». Esta obra de Jenofonte versaba sobre la correcta administración de la casa y del Estado. La economía nacía así como una ciencia más de análisis del comportamiento humano. Y esto último es significativo, puesto que, como veremos a lo largo de este artículo, la economía es un producto de la sociedad, es inherentemente social, y por tanto, conocer la economía de una sociedad a estudio, es acercarse a una comprensión más exacta de las sociedades pasadas.

\section{Hacia una comprensión del termino economía y su función de análisis del comporta- miento humano.}

Es una constante en el pensamiento económico actual señalar que el origen de la economía como disciplina científica está en el problema de la escasez. Parece evidente que sólo es posible economizar aquello que es escaso, los alimentos, el agua, etc. El problema fundamental de un estudio económico estriba en averiguar como una sociedad satisface sus necesidades ${ }^{1}$ sujeto a las restricciones impuestas por sus posibilidades reales de consumo, es decir, por el límite que imponen unos recursos escasos. Lo importante será pues en economía la «elección», ya que fruto de las elecciones que realicen los individuos u otras entidades determinarán estas como se emplean los recursos de que se dispone. La economía es pues una ciencia social que estudia el problema de la «elección».

Existen diversas definiciones sobre el área de influencia de la economía. Podemos definir la economía, empleando cualquier manual de economía al uso, como

1 Nadie en economía habla de necesidades hoy en día. Aunque se considera un concepto válido, se lo considera completamente subjetivo y ajeno/exógeno al problema de cualquier agente económico. El interés en economía actualmente radica en conocer como un individuo satisface sus preferencias - maximiza su utilidad u otro objetivo individual - sujeto a las restricciones impuestas por sus posibilidades reales de consumo. La coordinación de múltiples individuos con preferencias sobre múltiples productos y acceso a múltiples mercados sería el objetivo de la macroeconomía. 
la ciencia que estudia la conducta social referente a la producción, intercambio y distribución de bienes y servicios con recursos escasos, para la satisfacción de las necesidades humanas. En palabras del Prof. Samuelson, «el estudio de la manera en que las sociedades utilizan los recursos escasos para producir mercancías valiosas y distribuirlas entre los diferentes grupos». La economía, pues, trata de la conducta de los hombres, no de la acumulación de bienes, ni siquiera de la conducta de los hombres acerca de la acumulación de bienes (Argandoña et alii, 1983).

Las necesidades humanas son múltiples y susceptibles de infinito desarrollo ${ }^{2}$. En términos generales si el hombre ha conseguido satisfacer sus necesidades más inmediatas y más elementales (alimentarse, abrigarse, vivienda...), después querrá satisfacer otra serie de necesidades (cultura, servicios, etc...). El carácter ilimitado de las necesidades y el carácter limitado de los medios para satisfacer necesidades hacen que las acciones de los hombres comporten necesariamente elecciones. Como diría Stiglitz (1995: 28) «El término escasez ocupa un lugar destacado en economía: las elecciones son importantes porque los recursos son escasos».

Esta postura en cuanto a la interpretación de la economía ha sido duramente criticada desde otras posiciones teóricas y más en concreto desde el campo de la arqueología que es donde estriba nuestro interés (Risch, 2002a y 2002b, Briz y Godino 2002 , etc.). Se critica que el pensamiento económico actual ha generado una oposición entre la sociedad y la economía que anula a la primera para que se puedan así cumplir las leyes que establece la segunda. La crítica llega a su máxima expresión cuando se afirma que la mayor parte de la teoría económica moderna ha perdido su utilidad para una investigación histórico-social (Risch, 2002a: 6). Esta disociación entre pensamiento económico actual y sociedad no tiene sentido en tanto en cuanto todo análisis económico lo que pretende es describir el comportamiento de una serie de individuos interrelacionados en sociedad para satisfacer sus preferencias. Es decir, la una sin la otra no es nada. Risch critica más adelante en su texto que fuera del marco que delimitan la escasez y los precios, la teoría económica actual no posee «categorías de análisis», lo que imposibilita su aplicación al estudio en arqueología, ya que el concepto de escasez conlleva la exclusión de buena parte de los productos sociales del análisis económico. Esta interpretación obvia que la economía es capaz de estudiar una amplia gama de asuntos más allá del ideal de mercados competitivos.

Quienes hacen este tipo de críticas al pensamiento económico actual soslayan que la economía es una ciencia aplicada a la que se le exige constantemente respuestas

2 No necesariamente las necesidades tienen porque ser ilimitadas, los individuos tienen preferencias sobre diferentes bienes y servicios. Es el principio de «cuanto más mejor» que basa los supuestos sobre el comportamiento humano en que se fundamenta la economía y que es el que justifica que un incremento en los medios disponibles se traduzca en un incremento del consumo de bienes y servicios. 
para problemas de actualidad. Los economistas se encuentran con la obligación de facilitar tales respuestas y ello implica que muchas aplicaciones de la ciencia económica se hayan circunscrito al estudio del comportamiento económico en el último siglo, lo que no imposibilita que las mismas técnicas e ideas no tengan aplicación válida para dar respuesta a otros problemas económicos en otros períodos históricos. Además los economistas trabajan mediante la generación de modelos económicos a través de la realización de abstracciones del mundo real (mucho más complejo) pero que intenta reflejar las características esenciales de la economía a estudio. Ningún modelo es capaz de describir la realidad con una exactitud meridiana, así que lo importante es si el modelo planteado puede ser considerado un buen modelo para interpretar la realidad a estudio (Nicholson, 2004). En otras palabras, no se puede rechazar la teoría económica moderna simplemente porque desarrolle modelos y abstracciones, puesto que al fin y al cabo, en nuestra labor de historiadores realizamos lo mismo, modelos y abstracciones. La contrastación de estos modelos con la información disponible para comprobar su validez será el método de refutar o avalar a un modelo económico como efectivo para la interpretación de una economía dada a estudio. La dificultad estribaría en ajustar los métodos que postula el pensamiento económico actual a las posibilidades del registro arqueológico.

Consideramos prioritaria la apertura de caminos que fomenten la colaboración entre historiadores y economistas, como ya se ha hecho con otras disciplinas (química, geología, etc.), ahondando en la necesaria interdisciplinariedad. Es un hecho, con todo lo negativo que ello comporta para nuestras investigaciones que son sociales igual que las que realizan los economistas, que existe actualmente una disociación entre ambas ramas de investigación. Nos hemos estancado en el pensamiento económico de finales del siglo XIX y principios del siglo XX como sustento a nuestras investigaciones históricas, cuando muchos de los conceptos que ahora nosotros usamos, pretendidamente como fundamentales para los estudios de economía en historia, están superados dentro de la ciencia económica. Esto debería llevarnos a una profunda reflexión de nuestros planteamientos, e igual que nos estamos abriendo a las nuevas posibilidades que otras ciencias nos aportan (la geografía con los SIG - Sistemas de Información Geográfica - son un clarísimo ejemplo de lo que quiero expresar), lo mismo deberíamos hacer con la ciencia económica. Simplemente debemos hacer algo tan sencillo como plantear a los economistas cuales son nuestras preguntas y cual la información de que disponemos, para que estos nos puedan facilitar las mejores herramientas para estudiar las economías pretéritas.

\section{Factores básicos determinantes del crecimiento económico}

A la hora de realizar un estudio económico en que nos planteemos la evolución 
de una economía, hay una serie de aspectos cuyo análisis es necesario considerar por su impacto en el crecimiento y desarrollo de la misma. Entre ellos queremos destacar en primer lugar aquellos aspectos que se han venido considerando como los factores más importantes que fomentan el aumento de la producción y de la productividad ${ }^{3}$. Se han considerado tradicionalmente tres los factores que potencian el crecimiento de una economía:

- la población (mano de obra)

- el progreso técnico (que influye principalmente en el incremento de la productividad)

- el aumento de la capacidad productiva ${ }^{4}$.

El factor población, considerando el crecimiento demográfico, actúa en dos direcciones opuestas sobre el desarrollo: como propulsora de la actividad productiva y como reductora del acceso al alimento por habitante. Convendría aquí realizar una breve descripción de la teoría sobre la población de Malthus que resulta sumamente interesante para nuestro estudio; según este autor, al estudiar a las sociedades agrarias pre-capitalistas, observó que la producción tendía a crecer en forma lineal conforme se aumentaban los factores de producción disponibles, entre ellos tierra y trabajo, mientras que la población tendía a crecer en forma exponencial. Una de las razones que argumentaba para justificar el crecimiento lineal de la producción era que el incremento de la producción obligaba a las sociedades agrícolas a poner en producción tierras marginales cuya calidad era inferior. El resultado es que la productividad de esas tierras era menor, pero los requisitos de trabajo seguían siendo los mismos (o incluso mayores). A esto es a lo que Malthus implícitamente y Marx explícitamente se referían como la ley de «rendimientos marginales». Las consecuencias que ambos autores clásicos derivaban eran radicalmente opuestas. La interpretación de Malthus versaba en que el crecimiento exponencial de la población implicaba que la cantidad de producto agrícola per cápita se redujese paulatinamente, lo que producía hambrunas, provocaba guerras, enfermedades, etc. La teoría de Marx es que esta ley era universalmente válida y provocaba algo más que hambrunas, puesto que fomentaba la aparición de desigualdades sociales y la opresión en función de quienes ostentasen

3 Hay que tener en cuenta que los factores expuestos lo son desde el lado de la oferta, debiendo considerarse el impacto que tiene la demanda social (o demanda agregada) sobre la producción de bienes.

4 Entiéndase por aumento de capacidad productiva cuando una economía que opera en el óptimo de su frontera de posibilidades de producción (FPP) es capaz de desplazar la curva que representa su FPP a la derecha a través de una serie de factores, que serán desarrollados en el texto más adelante, con lo que la producción máxima que puede alcanzar una economía se ve incrementada. 
la propiedad de los medios de producción. En resumidas cuentas, según Marx la reducción del producto per cápita no afectaría a todos los individuos por igual; habría clases privilegiadas y clases sometidas.

Creemos que el aumento de la población en sí no constituye un impedimento al desarrollo, siempre y cuando la generación de alimentos siga un ritmo más acelerado que el de la población. El crecimiento demográfico podría venir dado por una mejora de las condiciones alimenticias resultado de mejoras en la producción que unido a algunas mejoras en las condiciones de higiene podría haber resultado en un incremento de la esperanza de vida. Este aumento en la esperanza de vida significaría la posibilidad de más embarazos, suponiendo un incremento de la población, aunque la tasa de mortalidad en infantes se mantuviese constante. También el incremento poblacional podría estar relacionado con conflictos entre distintos grupos que condujera al aumento demográfico para entrar en competencia con otros territorios, siendo la población uno de los instrumentos mediante los cuales se tratase de sobrepasar el equilibrio de fuerzas del otro. La idea de un aumento poblacional durante la Edad del Bronce en tierras valencianas (por poner un ejemplo) con respecto a etapas anteriores parece ser un hecho aceptado por la mayoría de autores, aunque matizando este incremento poblacional en base al pequeño tamaño de los poblados y la corta existencia de los mismos (Tarradell, 1963; Martí Oliver, 1983; Hernández Pérez, 1985; Gil-Mascarell, 1992; De Pedro, 1994; Jover Maestre, 1999; etc).

Hemos de señalar que el crecimiento demográfico repercute en un aumento de la mano de obra disponible para el trabajo5. Esto podría repercutir en un aumento de la productividad por cuanto permitirá mejorar la eficacia del trabajo, entre otros aspectos.

Siendo lo anteriormente dicho de relevancia, parece en realidad mucho más importante el impacto de las características tecnológicas. El estudio de la mayoría de herramientas empleadas, fabricadas en piedra y madera principalmente (aunque el reflejo arqueológico de las segundas sea difícil de observar por cuestiones de conservación de dicha materia prima), su cambio respecto a etapas anteriores y el análisis de su impacto en los procesos de producción es una interesante vía de estudio económico que deberemos explorar con exhaustividad por cuanto que es un elemento que incide directamente y de manera muy significativa en el aumento de la productividad $^{6}$. Se debe tener en cuenta que la demanda condiciona tanto el sistema de pro-

5 Risch afirma la imposibilidad existente en arqueología para abordar el estudio del trabajo, sólo pudiendo acercarse al mismo de forma indirecta a través de los Medios de Producción utilizados en la transmisión de la fuerza humana y de los productos resultantes (2002b: 18).

6 Productividad significa producir más con el mismo consumo de recursos, o también producir lo mismo pero con un consumo menor de los recursos, pudiendo emplearse los recursos economizados para la producción de otros bienes (Kanawaty, 1981). 
ducción agraria como el progreso tecnológico, consistiendo este en una combinación de especialización y concentración de herramientas y procesos que permiten una mayor capacidad de producción (López Gálvez et alii, 2000). En algunos casos, la existencia de productos metálicos puede no estar reflejando la existencia de avances tecnológicos para la producción, sino simplemente el acceso de algunos miembros de una comunidad a productos diferenciales con un mero carácter simbólico, que no funcional.

El aumento de la producción agrícola, ganadera y artesanal - aunque no tiene porque darse en todas a la vez-, puede deberse a varias razones: la mejora técnica en el proceso de producción que permite producir más con los mismos recursos, el aumento del área cultivada, el incremento de las cabezas de ganado o quizá por el crecimiento en la mano de obra disponible para el trabajo lo cual puede deberse a un incremento de la población ${ }^{7}$. Vinculado a esto habrá que conocer cual es el proceso de producción, para poder estimar el verdadero impacto de las dos variables expuestas sobre el aumento de la capacidad productiva. No debemos en este punto olvidar el efecto que la ley de la entropía introduce en el ciclo agrícola, puesto que esta ley explica la imposibilidad de que crezca cereal todos los años en el mismo trozo de terreno (cultivar significa explotar el suelo). Esta ley explica la dependencia que tiene la vida terrestre del sol, hasta el punto que se ha incorporado al patrón reproductivo tanto de las especies vegetales como de las animales. Es un hecho conocido que tan sólo en determinados períodos del año se puede realizar el cultivo de los cereales - por ejemplo-, o que sea en primavera el período de nacimiento de los corderos - por poner otro ejemplo- . La energía con que el sol llega a cada lugar de la tierra determina el ritmo cíclico de reproducción en cada zona. Por ello deberemos tener en cuenta la existencia o no de prácticas de abonado/estercolado que permiten ralentizar la degradación de los suelos (López Gálvez et alii, 2000) y la probable puesta en explotación de nuevas tierras una vez que las que se ha estado explotando han dejado de ser productivas, con lo que podemos encontrarnos ante la explotación de tierras con menores capacidades agrícolas. Además la existencia de períodos en que es imposible el cultivo de las especies vegetales determinará que el tiempo en que la actividad agrícola sea imposible de realizar se destine a otras actividades económicas (metalurgia, cestería, tejido, alfarería,...).

7 Todo ello dependerá, como no, de las características del proceso productivo, ya que es posible que dependiendo de la producción sea necesario esperar a que se produzca un incremento del área cultivada (manteniendo un mismo nivel de fertilidad constante) para que realmente la producción crezca. 


\section{El enriquecimiento como base de la división social}

Otro aspecto de interés sería el analizar como se produce el enriquecimiento de la sociedad o de algunos miembros de la misma y como se podría generar una división social en base a ese enriquecimiento. Son varias las razones que podemos esgrimir para buscar en ellas la respuesta a esta cuestión.

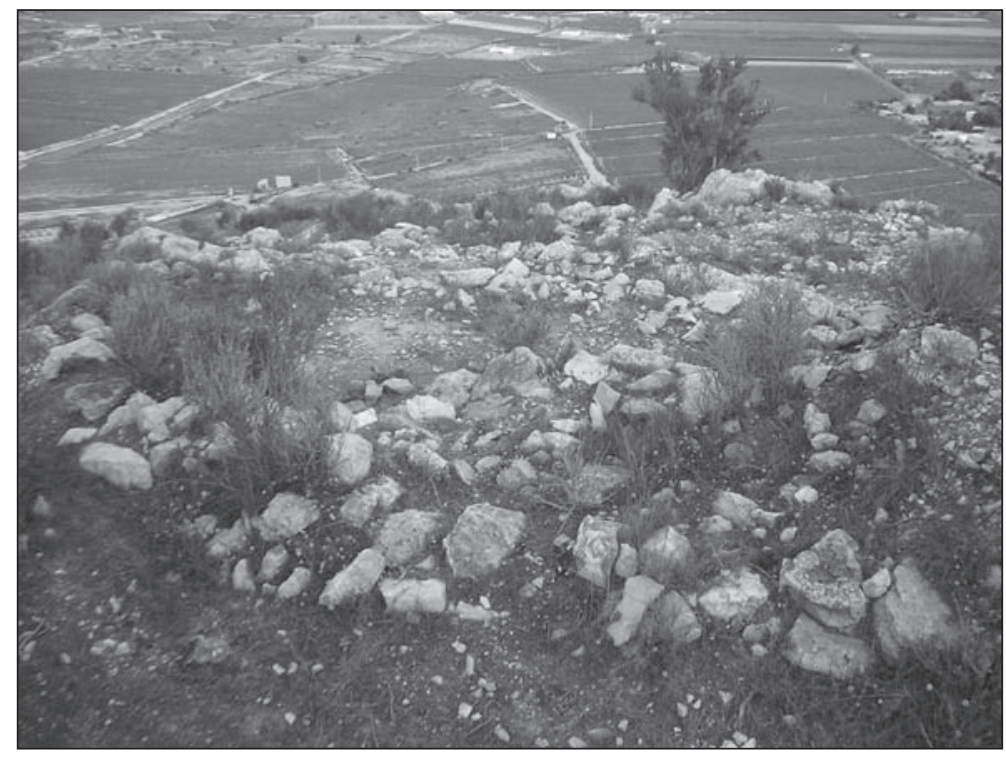

Imagen de un molino del yacimiento de El Calafuch (Monovar, Alicante). Edad del Bronce.

El aumento de la producción agrícola sería un primer aspecto a tratar para buscar en él una de las causas del enriquecimiento de algunos sectores de la sociedad. Para dar mejor respuesta a esta cuestión deberemos plantearnos el problema de la propiedad de la tierra, ya que dependiendo de la fertilidad de las tierras que se cultiven la productividad que obtenga de sus tierras cada poblado variará (o quien sea que explote una determinada parcela de tierra), siendo también muy importante el elemento tecnológico por su notable incidencia en el aumento de la productividad. No olvidemos algo básico y fundamental en economía, y es que el medio de conseguir más con los mismos recursos es la especialización y el comercio. Un aumento de la productividad lleva a una elevación del nivel de vida (Kanawaty, 1981).

Debemos plantearnos también el diferente o igual acceso de cada individuo a la propiedad dentro de un mismo poblado, ya que en función del acceso que se tenga a la propiedad de la tierra se estarán generando unas desigualdades sociales (Gilman, 
1997: 82). «To understand an economy, the first question to be resolved is the nature of land tenure (ownership)» (Earle, 2002: 9). Se ha señalado que en las sociedades prehistóricas o primitivas un individuo accede a la propiedad en base a su pertenencia a un grupo, lo que implica que la posesión de la tierra es del conjunto de los individuos, de la sociedad, y que por tanto hablemos de propiedad comunal (Godelier, 1981).

Una propuesta de interpretación del registro arqueológico interesante a la hora de intentar acercarnos a los sistemas de propiedad es la expuesta por Gilman de esquema evolucionista por la cual «a medida que una producción resulta intensificada, la propiedad privada abarca una gama de objetos más amplia» (1997: 85); siendo importante pues indagar la intensificación o no, en el consumo de bienes que han quedado fosilizados en el registro arqueológico, y también en el consumo diferencial que pueda o no percibirse dentro de las áreas habitacionales excavadas (o en los continentes funerarios), siendo para ello muy necesario el disponer de importantes series estratigráficas y de estudios microespaciales.

Timothy K. Earle ha desarrollado una propuesta de interpretación de la propiedad en Prehistoria (2000) intentando evaluar una serie de cuestiones que pueden tener reflejo en el registro arqueológico, basado en cuatro aspectos:

1.- El trabajo destinado a la elaboración de objetos específicos y la intensificación en la producción son una medida indirecta para sacar conclusiones en torno a la propiedad.

2.- El desarrollo de la guerra es una causa de la intensificación agrícola y del incremento del valor de la tierra, y debe ponerse en relación con unos emergentes derechos sobre la propiedad. La guerra tendría que ponerse asimismo en relación con conflictos surgidos sobre derechos de propiedad entre grupos en los que no existe un sistema legal sobre la misma.

3.- La distribución de los asentamientos en el territorio pueden reflejar pautas de posesión de la tierra. Los estudios de territorio no definen por si mismos derechos de propiedad, pero si que permiten asociar los recursos con los grupos humanos que los explotan. Cambios en los patrones de asentamiento nos están indicando que se ha producido una transformación social y, asociada a ello, cambios en las formas de propiedad. En las excavaciones, la diferente distribución de los objetos de valor dentro de un poblado, deben estar reflejando diferentes accesos a la propiedad.

4.- El marcar o delimitar un terreno o un objeto es la prueba más directa de los derechos de propiedad individual o colectiva. La intensificación del uso de la tierra esta asociado con derechos de posesión de la tierra más explícitos que son marcados mediante construcciones físicas - vallados-. 
Relacionado con todo esto y quizá mucho más importante, será el analizar como se reparte entre los miembros de una misma comunidad los bienes producidos, lo que nos podrá dar una idea clara de la organización social y de la existencia o no de distintos grupos sociales dentro de una comunidad. Pero como ya hemos mencionado para ello deberíamos contar con series estratigráficas y análisis microespaciales, cuya casi total ausencia en las publicaciones de las excavaciones, hasta al menos finales de los años 90, suponen un lastre a la investigación.

Una cuestión de relevante interés para nuestro estudio será el uso del plusproducto o en su caso excedente ${ }^{8}$ por parte del grupo social que integre cada poblado ya que la disponibilidad de este para comerciar con otras áreas o poblados limítrofes supone un hecho diferencial que permite el enriquecimiento del grupo o de parte del mismo (dependiendo del uso del plusproducto o el excedente entendiéndolo en los términos propuestos por la Arqueología Social Latinoamericana). Debemos tener en cuenta que el comercio permite especializarse más y que la especialización redunda en un aumento de la productividad (Stiglitz, 1995). Parece obvio pues afirmar que la especialización y el intercambio contribuyen en gran medida a que aumente la eficiencia, tanto en la producción como en el producto elaborado. El intercambio sería el factor clave por cuanto que aumenta los beneficios dando eficiencia a la producción:

Intercambio $=$ Especialización $=$ Eficiencia.

Pero no tienen porque ser estos los únicos medios de enriquecimiento de la población. Hay muchos factores que pueden influir y uno de ellos podría ser el surgimiento de la industria metalúrgica y la necesidad derivada de profundizar en los principios de división del trabajo. Las familias al no poder producir todo tienen la necesidad de especializarse ${ }^{9}$ cuando sus necesidades subsistenciales básicas están aseguradas (producción de alimentos), o por el contrario, por el hecho de que una de las virtudes de la especialización es que las necesidades básicas se pueden satisfacer a través del comercio. La diferenciación en el trabajo puede conducir al enriquecimiento de determinados grupos sociales y ello podría llevar a la aparición de distintas clases sociales. La producción de la industria metalúrgica tiene un valor superior al de la producción agrícola-ganadera, estas diferencias pueden explicar la tendencia al enriquecimiento

8 Según plantea Javier Jover (1999) siguiendo a Luis Felipe Bate, hablamos de excedente cuando el plusproducto producido por los productores directos les es enajenado por parte de un grupo dominante.

9 Siguiendo a Joseph E. Stiglitz (1995) la especialización es un factor que puede influir en el aumento de la productividad en base a tres razones: 1 . El trabajador no perderá tiempo en cambiar de tarea. 2. Los trabajadores que repiten en una tarea se vuelven mucho más cualificados en la misma y 3. La especialización crea un clima favorable para la innovación. 
de determinadas clases sobre todo si en particular lo relacionamos con la escasez de los factores productivos ${ }^{10}$ en la metalurgia (materias primas) que hace que este incremente su valor, aunque no necesariamente.

\section{Preguntas clave para un estudio económico y del área a estudio en particular}

Toda sociedad humana, ya se trate de una sociedad capitalista como si se trata de una pequeña comunidad aislada, debe afrontar cuatro problemas económicos básicos que se encuentran relacionados entre sí y dar respuesta a los mismos. Siguiendo a Joseph E. Stiglitz (1995) estos cuatro problemas serían:

¿Qué bienes y servicios producir y en que cantidades?

¿Cómo van a producirse estos bienes y servicios?

¿Para quién van a producirse estos bienes y servicios?

¿Quién toma las decisiones económicas y mediante qué procedimiento?

Estos problemas son comunes a todas las economías pero cada sociedad responde a ellos de forma diferente. El estudio de los sistemas económicos, entendiendo por tal la forma de organizarse que tiene una sociedad para satisfacer sus necesidades y las de sus individuos, nos muestran los mecanismos que pueden utilizarse para dar respuesta a las anteriores cuestiones.

Antes de responder que tipo de organización económica predominaba en cada momento y lugar habrá que responder a una serie de preguntas que nos permitan aportar luz a los ya mencionados anteriormente cuatro problemas básicos de todo análisis económico. Las preguntas que debemos hacernos son:

¿Qué sector económico es dominante?

¿El sistema es autárquico o existe comercio entre distintas zonas?

¿Cómo se producen los intercambios de recursos y de qué manera se ven condicionados?

¿La economía depende del trueque o existe alguna evidencia de que se usaba algún elemento como medio de intercambio o depósito de valor?

¿Qué factores determinan el crecimiento económico?

¿Qué indica el registro arqueológico acerca de las posibilidades de producción de la economía o de la organización social?

¿Qué tipo de organización política existe, son poblados autónomos, dependen unos de otros?

10 Son tres los factores productivos a considerar. Por un lado la tierra en su sentido más amplio, desde la tierra cultivable hasta las materias primas que ofrece - minerales, agua, luz que recibe...-_; por otro lado el trabajo considerando como factor de producción tanto las capacidades físicas como las intelectuales, aplicadas a la producción de bienes y servicios; y por último el capital refiriéndose este término al equipo y materiales empleados en el proceso productivo (arado, hoces, hachas...). 
¿Cómo se contribuye a la defensa y a la provisión de otros bienes públicos en caso de ser necesaria?

Siguiendo a Robert Chapman, para estudiar la complejización deberemos mostrar especial atención en el análisis de las siguientes variables: intensificación, innovación tecnológica, escala del sistema, interacción e integración. Todo ello deberá ser confrontado con el registro arqueológico y las posibilidades de interpretación que este permita (Chapman, 1991: 41).

Estas son algunas de las cuestiones cuya respuesta nos orientará a la hora de interpretar el sistema económico de la sociedad dada a estudio. Además de otras tantas que podamos plantear debido a las particularidades que observemos en las distintas sociedades. La economía tiene un fuerte impacto en lo social como se ha podido observar en estas líneas, con lo que conociendo el tipo de economía y todas las implicaciones de esta, se puede llegar a establecer hipótesis sobre el desarrollo social de las entidades a estudio. Pero todo ello estará siempre sujeto a la calidad del registro arqueológico y a las posibilidades de interpretación que este permita.

Las deficiencias del registro arqueológico y las dificultades para inferir aspectos económico-sociales

La tarea de la investigación arqueológica en palabras de Patricia Fournier (1994) es «la explicación de procesos, de las similitudes y diferencias sociales en las dimensiones temporal y espacial, evidenciadas en las manifestaciones materiales de la conducta humana, es decir, en la cultura material o el dato arqueológico». Siguiendo a Sonia Gutiérrez (1997: 27) «la arqueología aspira a explicar de forma científica problemas históricos previamente planteados, a partir de la recuperación y el estudio de los restos materiales de las sociedades del pasado». Para el estudio de sociedades que no dejaron un registro escrito, que podríamos definir como arqueología prehistórica (Gutiérrez Lloret, 1997: 40) la arqueología se convierte en el único método para poder realizar inferencias en torno a la misma. Como diría Jordi Estevez (1981: 297) «el material arqueológico es el reflejo, el único, de todas las actividades y de la estructura social y económica de las comunidades prehistóricas», existiendo pues para el estudio de la prehistoria lo que se ha denominado «unicidad de sus fuentes» (Gutiérrez Lloret, 1997: 176), con las limitaciones que el registro arqueológico impone a cualquier investigación, siendo quizá las limitaciones mas destacables las siguientes:

- No se conserva la totalidad de los materiales empleados por las sociedades a estudio.

- La formación del contexto arqueológico influye en gran medida en la información que es posible recuperar. 
- Los procesos de excavación, dependiendo de la rigurosidad de los mismos, obtendrán mayor o menor información del registro arqueológico. Las excavaciones de principios del siglo XX, o en momentos anteriores, en su mayoría no cuentan con el mínimo de rigurosidad requerido en la actualidad.

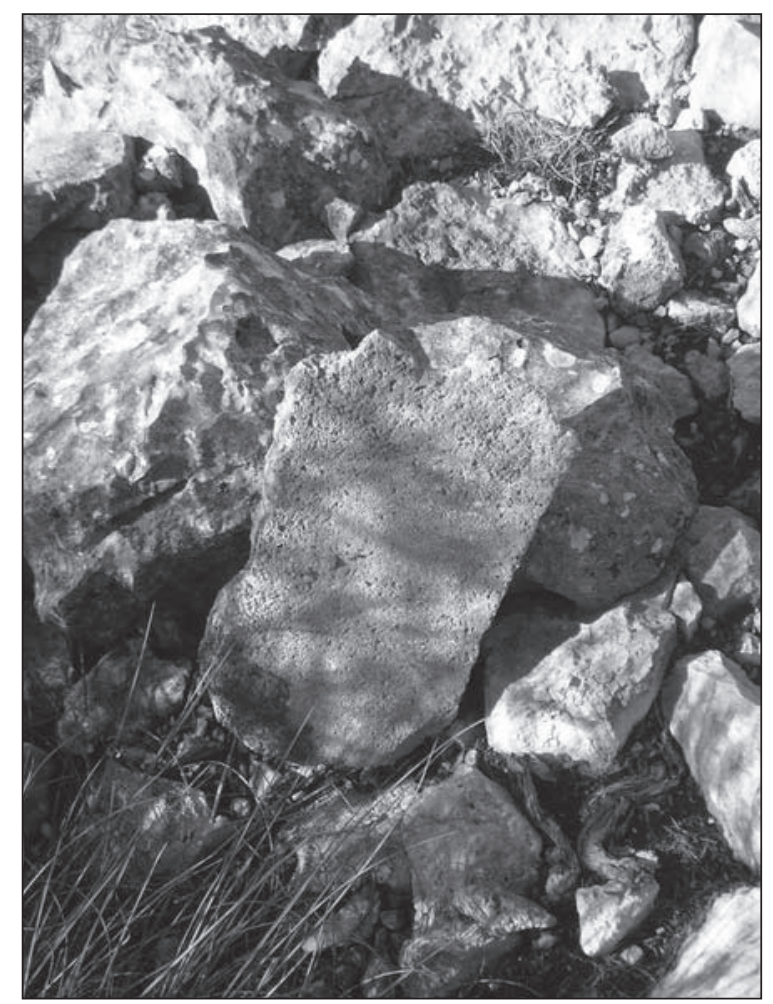

Fotografía del área excavada en el yacimiento de la Horna (Aspe, Alicante). Edad del Bronce

Uno de los problemas con los que se encuentra cualquier estudio arqueológico que pretenda realizar inferencias en torno a la economía de una sociedad dada es que tan sólo dejan huella directa en el registro arqueológico los medios de trabajo ${ }^{11}$ y los productos (Risch, 2002a y 2002b), a lo que podríamos añadir las áreas de trabajo y que es posible de determinar en base a los materiales presentes y al estudio microespacial de los mismos dentro de la unidad habitacional en la que han sido excavados.

11 Los medios de trabajo, los útiles o herramientas, son tanto productos como útiles que participan en nuevas acciones económicas (Risch, 2002b). 
Con todo ello, deberemos realizar un estudio económico intentando sustentarlo en la información que disponemos, e intentando comprobar en el registro si los presupuestos sobre crecimiento económico y aumento de la riqueza expuestos en este apartado encuentran su correlato material que los avale o refute, para poder realizar posteriormente conclusiones sobre la economía y la sociedad de los grupos centran nuestras investigaciones.

\section{Bibliografía}

APARICIO PÉREZ, J. «Avance al estudio económico de la Edad del Bronce Valenciano», I Congreso de historia del País Valenciano, Valencia, 1971, pp. 209-214.

- Estudio económico y social de la Edad del Bronce Valenciano. Ayuntamiento de Valencia, Valencia. 1976.

BANTON, M.(ed). Antropología Social de las Sociedades Complejas. Ed. Alianza, Madrid. 1980.

BELTRÁN MARTÍBEZ, M., HERNÁNDEZ PÉREZ, MS. y BERNAT MARTÍ, O. «Del Epipaleolítico a la Edad del Bronce en el País Valenciano: Tradiciones culturales, intercambios y procesos de transformación», Actas del XXIII Congreso Nacional de Arqueología, pp. 21-30.

BRANSON, W.H. y LITVACK, J.M. Macroeconomía. Harla, México. 1979.

BRIZ I GODINO, B. «Producción y consumo», BAR, 1073, Oxford, 2002. pp. 43-51.

CARNEIRO, R. «The chiefdom: precursor of the state», en JONES, G.D y KAUTZ, R.R. (eds): The transition to the statehood in the New World. University press. Cambridge. 1981.

CASTRO, P.V., LULL, V., MICÓ, R. y RIHUETE, C. «La Prehistoria Reciente en el Sudeste de la Península Ibérica. Dimensión socioeconómica de las prácticas funerarias», Arqueología da Morte na Península Ibérica desde as orixes ata o medievo, 3, Xinzo de Limia. 1995, pp. 127-167.

CHAPMAN, R.W. La formación de las sociedades complejas: El Sureste de la Península Ibérica en el marco del Mediterráneo Occidental. Ed. Crítica. Barcelona. 1991.

CONTRERAS, J. «La antropología de las Sociedades Complejas», en FRIGOLÉ, J. et alii. Antropología hoy. Una introducción a la Antropología Cultural. Ed. Teide. Barcelona. 1983.

CORRAL CAÑÓN, M. y RUBIO DE MIGUEL, I., «El asentamiento humano como indicador del cambio cultural. El caso de la región valenciana.», Cupuam, 15, Madrid. 1988, pp. 11-35.

DE PEDRO MICHÓ, MJ. «La Edad del Bronce en el País Valenciano: Estado de la cuestión», Saguntum, Valencia, 1992, pp. 61-87.

DORNBUSCH, R., y FISCHER, S. Macroeconomía. Mc Graw hill, Madrid. 1994. 
EARLE, T.K. Economic and social organization of a complex Chiefdom: the Halalea district, Kaua'i, Hawaii. Anthropological Papers. Museum of Anthropology, University of Michigan, $\mathrm{N}^{\circ}$ 63. Ann Arbor, Michigan. 1978.

- «Chiefdoms in archaeological and ethnohistorical perspective», Annual Review of Anthropology, 16, Palo Alto. 1987.

— «The evolution of Chiefdoms», en Current Anthropology. 1989.

— «Archaeology, property, and prehistory», en Annu. Rev. Anthropol. 29. pp. 39-60. 2000.

- Bronze Age economics. The beginnings of political economies. Westview press, Colorado. 2002.

EARLE, T.K., y JOHNSON, A. W. La evolución de las sociedades humanas. Desde los grupos cazadores-recolectores al estado agrario. Ariel Historia, Barcelona. 2003.

ENGUIX, R. «Notas sobre economía del Bronce Valenciano». Saguntum (PLAV), 12. Sagunto. 1977, pp. 11-30.

FOUNIER, P. «Teoría y praxis de la arqueología social: la inferencia de procesos económicos con base en conjuntos artefactuales».

FRIED, M.H. The Evolution of Political Society: An Essay in Political Anthropology. Random House. New York. 1967.

FRIEDMAN, J. «Tribus, estados y trasformaciones», en BLOCH, M.(ed). Análisis marxista y antropología social. Ed. Anagrama. Barcelona. 1977.

GALEY, C.W. «The state of the state in Anthropology», Dialectical Anthropology, 9 nums 1, 4, New York. 1985.

GALEY, C.W. y PATTERSON, T.C. «State formation and uneven development», en GLEDHILL, J. et alii.(eds). State and society: the emergence and development of social hierarchy and political centralization. One World Archaeology Series number 4, Unwin Hyman, London. 1988.

GIBSON, D.B. y GESELOWITZ, M.N. «The evolution of complex society in Late Prehistoric Europe: toward a paradigm», en GIBSON, D.B. y GESELOWITZ, M.N.(eds). Tribe and polity in Late Prehistoric Europe. Plenum Press, London. 1988.

GIL-MASCARELL, M. «El Bronce Tardío y el Bronce Final en el País Valenciano», Monografías del Laboratorio de Arqueología de Valencia, I, Valencia, 1981, pp. 9-39.

- «El final de la Edad del Bronce: Estado actual de las investigaciones», en Arqueología del País Valenciano: Panorama y perspectivas, Alicante. 1985, pp. 141152.

- «La agricultura y la ganadería como vectores económicos del desarrollo del Bronce Valenciano», Saguntum, 25, Valencia, 1992, pp. 49-67. 
- «Algunas reflexiones sobre el Bronce Valenciano», Saguntum, Valencia, pp. 63-73. GIL MASCARELL, M. y ENGUIX, R. «La cultura del Bronce Valenciano: Estado actual de la investigación», Homenaje a Luis Siret, , 1986, pp. 418-424.

GILMAN, A. «El análisis de clase en la Prehistoria del Sureste», Trabajos de Prehistoria, 44, Madrid. 1987.

- «Como valorar los sistemas de propiedad a partir de datos arqueológicos», Trabajos de Prehistoria 54, n², 1997, pp.81-92.

GILMAN, A. y THORNES, J.B. El uso del suelo en la prehistoria del SE de España. Fundación J. March, Madrid. 1985.

GODELIER, M. Teoría Marxista de las Sociedades Precapitalistas. Ed. Estela, Barcelona. 1971.

GODELIER, M. La producción de Grandes Hombres. Poder y dominación masculina entre los Baruya de Nueva Guinea. Ed. Akal, Madrid. 1986.

GONZÁLEZ RUBIAL, A. «Etnoarqueología de la vivienda en África subsahariana: Aspectos simbólicos y sociales», Arqueoweb 3-2, Madrid. 2001.

GUTIÉRREZ LLORET, S., Arqueología. Introducción a la historia material de las sociedades del pasado. Ed. Universidad de Alicante. Alicante. 1997.

HAAS, J. The evolution of the Prehistoric State. Columbia University Press. New York. 1982.

HARDING, A.F. Sociedades europeas de la Edad del Bronce. Ariel Prehistoria, Barcelona. 2003.

HERNÁNDEZ PÉREZ, M. S. «Cueva de la Casa Colorá: un yacimiento Eneolítico en el Valle Medio del Vinalopó (Alicante)». Lucentum, I. Alicante. 1982, pp. 5-18.

- «La Metalurgia prehistórica en el Valle Medio del río Vinalopó (Alicante)», Lucentum, II, Alicante, 1983, pp. 17-42.

- «La Edad del Bronce en el País Valenciano: panorama y perspectivas», Arqueología del País Valenciano: panorama y perspectivas, Alicante, 1985, pp. 101-119.

JOVER MAESTRE, F.J. Una nueva lectura del bronce valenciano. Universidad de Alicante. Alicante. 1999.

- «La producción lítica de las entidades sociales de la Edad del Bronce», en ...Y acumularon Tesoros. Mil años de historia en nuestras tierras. CAM, Alicante. 2001, pp. 239-246.

JOVER MAESTRE, F.J. y LÓPEZ PADILLA, J.A. «Caracterización del patrón de asentamiento de la cuenca del río Vinalopó (Alicante) durante el II Milenio Ane», Actas del XXIV Congreso Nacional de Arqueología, Cartagena. 1997, pp. 241249.

- «Campesinado e historia. Consideraciones sobre las comunidades agropecuarias de la Edad del Bronce en el corredor del Vinalopó», APL, XXIII, Valencia, 1999. pp. 233-257. 
- «Una nueva propuesta del alcance espacial septentrional de las prácticas sociales argáricas», Congreso Nacional de Arqueología, Cartagena, 1994, pp. 275-287.

- «El Argar y el Bronce Valenciano. Reflexiones en torno al mundo funerario», Trabajos de Prehistoria 52, nº 1, 1995a, pp. 71-86.

- El poblamiento durante el II milenio A.C. en Villena (Alicante). Fundación Municipal, «José María Soler García». Villena. 1995b.

- «El Vinalopó. Gestión del territorio y de los espacios hídricos durante el II Milenio ANE», Congreso de Agua y Territorio, Elda, 1997a, pp. 163-187.

- Arqueología de la muerte. Prácticas funerarias en los límites del Argar. Universidad de Alicante, Alicante. 1997b.

- «2100 -1200 BC. Aportaciones al proceso histórico en la cuenca del río Vinalopó», en en La Edad del Bronce en Tierras Valencianas y zonas limítrofes, Hernández Péres, M.S. y Hernández Alcaraz, L. (eds). Villena. 2004, pp. 285-302.

- «La ocupación humana de la Cubeta de Villena: de los primeros grupos cazadores-recolectores a los origenes del iberismo», en Villena. Arqueología y Museo. Diputación de Alicante, Alicante. 2005, pp. 18-41.

- Barranco Tuerto y el proceso histórico durante el II milenio BC en el Corredor del Vinalopó. Vestigium I, Monografías del Museo Arqueológico de Villena. Villena. 2006.

KANAWATY, G. coord. Introducción al estudio del trabajo. Tercera Edición. Organización Internacional del Trabajo. Ginebra. 1981.

LULL, V. La «Cultura» de El Argar. Un modelo para el estudio de las formaciones económico sociales prehistóricas. Ed. Akal, Madrid. 1983.

MALTHUS, T. R. Ensayo sobre el principio de la población. Ed. F.C.E, México. 1986.

MARTÍ OLIVER, B. El nacimiento de la agricultura en el País Valenciano. Del Neolítico a la Edad del Bronce. Universitat de Valencia, Valencia. 1983.

MARX, K. y HOBSBAWM, E. Formaciones económicas precapitalistas. Ed. Crítica. Barcelona. 1979.

NICHOLSON, W. Teoría Microeconómica: principios básicos y ampliaciones. $8^{\mathrm{a}}$ Edición. Thomson editores. Madrid. 2004.

PEDRO MICHO, M.J. «La Edad del Bronce en el País Valenciano: estado de la cuestión», en ponencia presentada a Jornades d'Arqueologia Valenciana: 27 - 29 de gener de 1994, L'Alfàs del Pi (Alacant). 1994.

RISCH, R. «Análisis funcional y producción social: relación entre método arqueológico y teoría económica», BAR, 1073, Oxford, 2002, pp. 19-29.

- «Hacia una teoría económica en arqueología», en Recursos naturales, medios de producción y explotación social, Madrid, 2002, pp. 5-34.

SAHLINS, M. La economía del la Edad de Piedra. Ed. Akal. Madrid. 1983. 
SCHUMPETER, J.A. Historia del análisis económico. Ariel Economía, Barcelona. 1971.

STIGLITZ, J.E. Economía. Ariel Economía, Barcelona. 1995.

TARRADELL MATEU, M. El País Valenciano del Neolítico a la Iberización. Anales de la Universidad de Valencia, XXXVI. Valencia. 1962-1963.

- (ed). Actas de la I reunión de Historia de la economía antigua de la Península Ibérica. Universidad de Valencia, Valencia. 1971. 